\title{
Probables anotaciones autógrafas quevedianas complutenses ${ }^{1}$
}

\author{
Julio Vélez-Sainz \\ Instituto del Teatro de Madrid \\ Universidad Complutense de Madrid \\ Edif. D Desp. 01309 \\ Facultad de Filología \\ Ciudad Universitaria 28040 \\ jjvelez@filol.ucm.es
}

[La Perinola, (Issn: 1138-6363), 21, 2017, pp. 245-261]

DOI: 10.15581/017.21.245-261

Mucho ha llovido y mucho se ha avanzado en la confección de la librería de Francisco de Quevedo desde que hace cuarenta años Felipe Maldonado diera a conocer un manuscrito que contenía parte de su biblioteca en unos baúles que dejó al cuidado de Francisco de Oviedo². En el inventario, hecho en Madrid los días 18 y 19 de abril de 1646 en las casas de Francisco de Oviedo, Juan de Molina y el canónigo Guerrero, testamentarios del escritor, se reflejan y tasan los libros, los baúles y cofres donde están guardados, y otros objetos (armas, prendas de vestir o instrumentos de escritura). Desde entonces los estudios sobre la biblioteca de Quevedo cuentan ya con un buen número de referencias e ítems bibliográficos ${ }^{3}$. La más reciente edición del listado

1. Este artículo se enmarca dentro del proyecto de investigación «Base de datos y análisis de los rudimentos compositivos profesionales en la Edad de Orom, Estancias de movilidad de profesores e investigadores senior en centros extranjeros 2014, Ministerio de Educación, Refol. Prx14/00553 y de los objetivos investigadores de los proyectos TEAMAD (н 2015 / нuм-3366) у ртсе (ғFI2015-64799-р) del Instituto del Teatro de Madrid.

2. Maldonado, 1975. Se guarda este documento en el Archivo Histórico de Protocolos de Madrid (Prot. 8471, fols. 135r-142v) y es un inventario de algunos objetos y muchos libros que Quevedo dejó a su muerte a su sobrino y heredero Pedro de Alderete (o Aldrete) Quevedo y Villegas. Una parte de estos libros acabó en poder del Duque de Medinaceli que los hizo llevar a su palacio de Sanlúcar de Barrameda y otra parte pudo quedar en Madrid. La importante biblioteca de los Medinaceli, 1471 volúmenes, acabó siendo vendida al monasterio madrileño de San Martín y está recogida en varios manuscritos, uno de los cuales, el más completo de ellos, se guarda en la Real Academia de la Historia (sig.: 9/2009) en un catálogo conocido como Indice de San Martín.

3. Los más destacables son Alonso Veloso, 2010a y 2010b; Asensio, 1952; Rey (ed.), 1985, pp. 64-65; Blecua, (ed.), 1985, t. 1, p. 25; Cacho Casal, 2003 y 1998; Ettinghausen, 1964 y 1972, pp. 140-151; Fernández Galiano, 1945; Fernández González y Simões, 2011 y 2012, López Grigera, 2002; Martinengo, 1983; Pérez Cuenca, 2003 y 2004; Sánchez Laílla, 2003; Schwartz y Pérez Cuenca, 1999. 
y, por ende, la más completa, es la que realizara Javier Maldonado en 2011, quien complementó la lista ya referida de Felipe Maldonado hasta encontrar 152 ítems ${ }^{4}$ cuantificables de una biblioteca que, según informaciones del propio Quevedo, sumó 5000 ejemplares. Por otro lado, el grupo BIDIso ha iniciado el camino al intentar la reconstrucción de la Biblioteca «hipotética» de Francisco de Quevedo en la que se pretende completar la primera 5 .

Como complemento a los esfuerzos anteriores, podemos añadir ahora cuatro volúmenes -consignados pero no estudiados por Maldonado $^{6}$ - de la Biblioteca Histórica Marqués de Valdecilla de la Universidad Complutense. Estos libros no aparecen en la biblioteca "hipotética» de BIDIso ni en el inventario post mortem y la mayoría se refieren a ámbitos científicos. Todas estas obras tienen indudables ex libris que certifican que pertenecieron a la biblioteca de Quevedo. Los libros son los siguientes:

In hoc libro haec habentur Constantini Lascaris... De octo partibus or [ati] onis lib. I; eiusdem De constructione liber secundus; eiusdem De nomine [et] uerbo liber tertius; eiusdem De pronomine in omni idiomate loquendi, ac ut poetae utuntur opusculum... Cebetis tabula [et] graeca [et] latina... De literis graecis ac diphthongis... Abbreuiationes quibus ... graeci utuntur; Oratio Dominica [et] duplex salutatio ad Beatiss. Virginem; Euangelium diui Ioannis Euangelistae; Carmina aurea Pythagorae; Phocylidis poema ad bene beateque uiuendum; De idiomatibus linguarum tres tractatus Ioannis grammatici, Eustathii, Corinthi cum interpretatione latina; Introductio perbreuis ad hebraicam linguam (Venecia, Aldo Manucio, 1512) de Constantino Lascaris;

Ioannis de Vito... De causis nostrarum calamitatum et de morbis epidemicis: qui vulgabantur per totum regnum Neap. anno d[omi]ni 1600. Cum pronosticis vsq[ue] ad annum 1608 (Nápoles, Io. Iacobum Carlinum tipógrafo de la curia, 1602) de Giovanni de Vito;

De concordia liberi arbitrij, \& praedestinationis diuinae, liber unus (Lovaina, Rutgeri Reseij, 1537) de Johannes Driedo;

Libro della natura et virtù delle cose che nutriscono \& delle cose non naturali: con alcune osseruationi per conseruar la sanità É alcuni quesiti bellissimi da notare / raccolto... per M. Michel Sauonarola (Venecia, de Domenico \& Gio. Battista Guerra, 1576) de Johannes Michael Savonarola.

Como vemos, dos de ellos tratan de tema médico, en latín y en italiano: De causis nostrarum calamitatum et de morbis epidemicis de Giovanni de Vito y el Libro della natura et virtù delle cose che nutriscono de Johannes Michael Savonarola; de Giovanni de Vito también habría Quevedo leído un texto de adivinación sobre los eventos de 1608. Además encontramos un texto de teología -De concordia liberi arbitrij, E pradestinationis

4. Maldonado, 2011. El artículo incluye el índice completo y la edición del testamento.

5. Disponible en http://www.bidiso.es/IBso/FichaBibliotecaHipoteticaEscritor.do? $\mathrm{id}=$ qufrde 0002

6. Maldonado, 2012. 


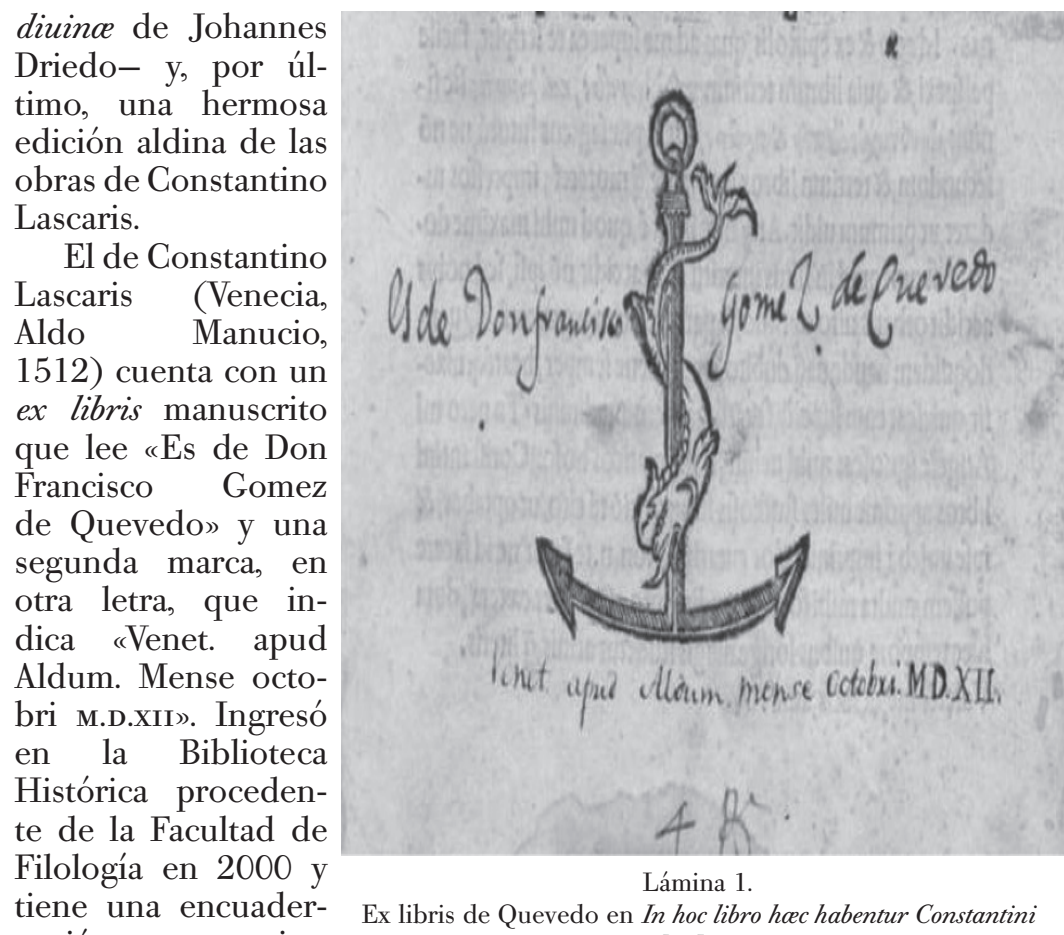
nación renacentista Lascaris... De octo partibus or[ati]onis lib. I de Constantino Lascaris, de piel sobre madera Biblioteca Histórica Marqués de Valdecilla, Universidad Complucon broches y cortes tense de Madrid, вн FLL Res.306

dorados. Se trata de una gramática muy común en el momento (en la misma biblioteca encontramos un segundo ejemplar con ex libris de la Biblioteca Complutense Ildefonsina ${ }^{7}$ ).

Este ejemplar es muy destacable en cuanto nos presenta un ejemplo físico del volumen del que partió Quevedo para realizar su propia traducción del griego de Focílides (en realidad, un seudo-Focílides). Quevedo publicó en 1635, en Madrid, y en la imprenta de María de Quiñones, su Epícteto y Phocílides en español con consonantes, libro del que se hicieron varias ediciones en los siglos XviI y xvıII. La traducción del seudo-Focílides ha merecido en nuestro siglo estudios de Castanien, de Crosby y Balcells ${ }^{8}$. Crosby mantiene que es «muy libre, en 594 endecasílabos con rima ${ }^{9}$ » e indica que posiblemente se sirviera de la famosa gramática griega De octo partibus orationis. En el ejemplar complutense

7. Ex libris ms. del Colegio Mayor de San Ildefonso de la Universidad de Alcalá. Ingresó en la Biblioteca Histórica procedente de la Facultad de Derecho en 2000. Encuadernación de pasta con super libros dorado del Cardenal Cisneros. Se trata del volumen BH DER 1413.

8. El artículo de Crosby incluye también la edición del texto.

9. Crosby, 1967, p. 176. 
se incorporaron, a modo de apéndice, los citados renglones métricos en griego que incluyen una versión latina entre líneas de la que a no dudar se valió Quevedo. A Balcells debemos la opinión verbal de María Rosa Lida que recogería Emilio Carilla ${ }^{10}$, a propósito de esta mantiene que don Francisco estaba muy lejos de conocer el griego con profundidad, y añadía: «la floja traducción de Píndaro, las versiones de Anacreonte y del seudo-Focílides servirán para testimoniar esa afirmación ${ }^{11}$ ».

El ejemplar complutense ratifica esta opinión. Se trata de un volumen no preparado para el trabajo en el que apenas encontramos marcas de lectura. En el prólogo hay tan solo unos subrayados en la página que destacar:

præceptorem tuum, a magno Alexandro dictum memorant, primus ter ante impressus, $q[u] e$ uita discederet ${ }^{12}$.

En el comienzo del cuaderno g, en un nuevo prólogo aparece con unas breves indicaciones:

"cognomento difficilis», "fuerunt, \& Priscianus», "quem latinum dicere Apollonium postumus», «ubi de constructione», «ad verbum fere tralatum a Prisciano Apollognium», “Scipio Carteromachus», «Nicolaus zudecus», «ut quibus nominibus græcas quasdam dictionis, latinas, ille dixisset, \& ipse $\underline{\text { dicerem }}^{13}$ )

y

"quatuor libri docti»), "Michaelis Sincele hierosolimitani», «doctus Theodorus ${ }^{14}$.

En el cuaderno $\mathrm{x}$, de nuevo, en un contexto prologal («Aldus lectori salutem») encontramos unos nuevos subrayados:

sæpiusque Terentiani illud sucurrerit, fronte exile negocium, \& dignum pueris putes: adgressis labor arduus, nec tractabile pondus est ${ }^{15}$.

En realidad, fuera de prólogos solo encontramos subrayados en un par de hojas, ambas en el cuaderno k:

10. Balcells, 1988, p. 68 .

11. Balcells, 1988, p. 38 .

12. Lascaris, De octo partibus, fol. alv.

13. Lascaris, De octo partibus, fol. g1r.

14. Lascaris, De octo partibus, fol. giir.

15. Lascaris, De octo partibus, fol. x1r. 
«Poesis autem homericissimi Cointi iam», «ppi Bessarion nicæas Cardinalis», «\& nunc i ultimis infeliciu[nt] græcoru[m] reuiuixit i[n] cognit ille orpheus sed appositum redeo ${ }^{16}$.

Todo parece señalar, en mi opinión, que se trata de un ejercicio de juventud en el que nuestro autor se encontraba en proceso de formación. No debemos olvidar, por otro lado, que Quevedo es un lector no académico por lo que, frente a un humanista profesional como Arias Montano, no tendría por qué leer en griego y sería lógico que se apoyara en la lectura del latín (que sí leería con facilidad). De hecho, pese a que fuera publicada en 1635, más que probablemente su traducción del seudo-Focilides se efectuara en sus años alcalaínos. Me atrevo a sugerir esta posibilidad a partir de varios factores. En primer lugar, la forma del ex libris de Quevedo incluye su primer apellido «Gómez», con el que firma sus trabajos de juventud. Luisa López Grigera (quien toma la noticia de Josette Riandière) indica que Quevedo a partir de 1613 deja de usar el apellido Gómez y se añade el Villegas ${ }^{17}$. Además, las marcas de lectura confirman que el texto apenas está trabajado: el latino muy poco y el griego nada.

El ejemplar de De causis nostrarum calamitatum et de morbis epidemicis de Giovanni de Vito (Nápoles, Giacomo Carlino, 1602) tiene un ex libris manuscrito en portada «D. francisco_de Quevedo-Villegas» con rúbrica al final de la firma. Ingresó en 2000 en la Biblioteca Histórica procedente de la Facultad de Medicina, cuya biblioteca estaba compuesta principalmente por los fondos del Instituto San Carlos. Las signaturas anteriores son v 85j, 613.1, y, anteriormente, la Biblioteca Histórica de H. Morejón, adquirida en 1876. No tiene marcas de lectura destacables. El volumen contiene una segunda parte (Liber secundus) titulada De prognosticis astrologicis futurarum nostrarum calamitatum ab anno 1600, usq. ad 1608 también por Giovanni de Vito cuya temática podría tener

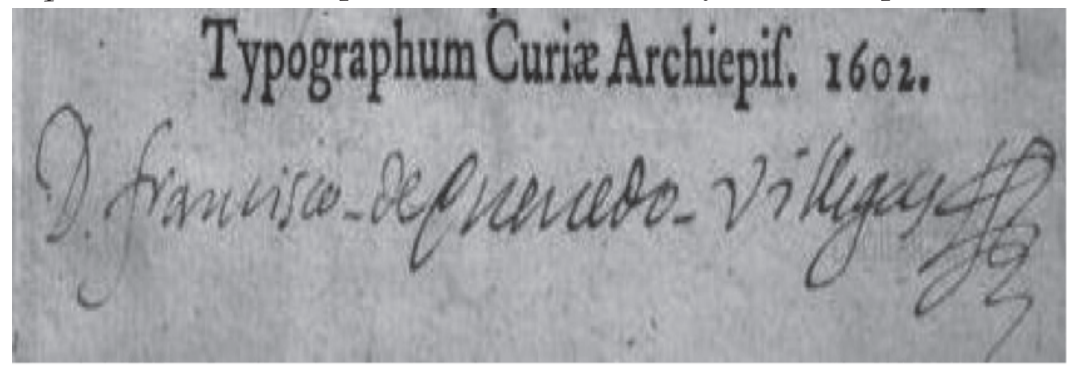

Lámina 2.

Ex libris de Quevedo en De causis nostrarum calamitatum et de morbis epidemicis de Giovanni De Vito, Biblioteca Histórica Marqués de Valdecilla, Universidad Complutense de Madrid, BH MED 3702

16. Lascaris, De octo partibus, fol. kvir.

17. López Grigera, 2002, p. 167. 
que ver con la obra quevediana, sobre todo con los Sueños en los que la profecía y la astrología tiene una indudable importancia como demostrara, hace ya muchos años, Alessandro Martinengo en La astrología en la obra de Quevedo.

Quevedo probablemente se hizo con este ejemplar derivado de la biblioteca del condestable Juan Fernández de Velasco, a quien está dedicado el volumen. Indica Jauralde Pou (1998, p. 217) en su magna biografía que Quevedo probablemente manejaría esta biblioteca en sus años vallisoletanos (1609-1610).

El texto de De concordia liberi arbitrij, E praedestinationis diuinae de Johannes Driedo (Lovaina, Rutgeri Reseij [Ruggiero Resei], 1537) también presenta varias anotaciones marginales en latín, comunes en nuestro autor. La letra de las anotaciones no corresponde con las características de la de Quevedo: se trata de una letra casi redonda, con trazos rectos, muy poco angulados, y donde ni encontramos ninguna de las marcas características de la péñola quevediana en las calas estudiadas para el Savonarola (vid. infra): la $g$, la $q$ minúsculas, la conjunción $d e$, etc. Se trata de un estilo muy cercano a la letra que anota el Antonio Lulio Valearis destacado por López Grigera y quizá se podría situar junto a las que la crítico considera como dudosas en su magno estudio: una mano de letra «libraria», que usaría Quevedo al escribir libros. En breve, las anotaciones a este volumen no se pueden atribuir con un mínimo de certeza, ni mucho menos de manera sistemática ${ }^{18}$.

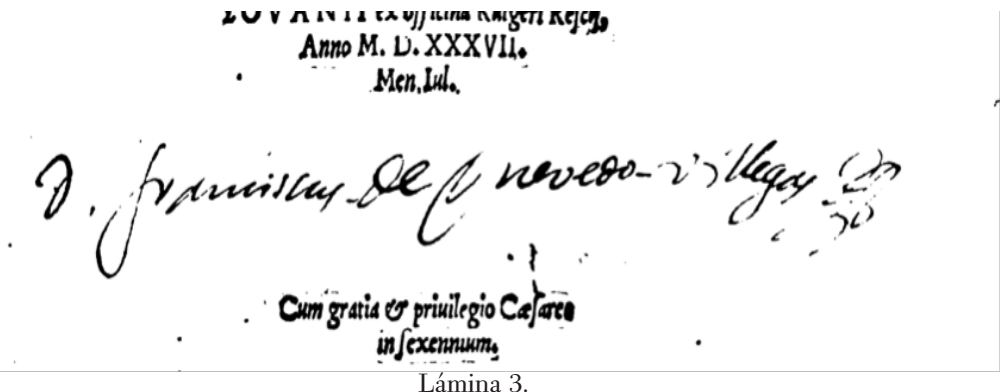

Ex libris de Quevedo en De concordia liberi arbitrï, E pradestinationis diuina de Johannes Driedo, Biblioteca Histórica Marqués de Valdecilla,

Universidad Complutense de Madrid, Bн FLL 6890

El Libro della natura et virtù delle cose che nutriscono $\mathcal{E}$ delle cose non naturali de Johannes Michael Savonarola (Venecia, Domenico \& Giovanni Battista Guerra, 1576) cuenta con otra anotación manuscrita en la portada «Don Francisco de Quevedo-Villegas» con rúbrica al final de la firma. Proviene de la Facultad de Medicina y cuenta con el sello del Colegio de San Carlos. Con encuadernación de pergamino, aparece encuadernada en un volumen facticio junto a otras dos obras de

18. López Grigera, 2002, pp. 186-187. Se encuentra en вN 3 / 59459. 
tema médico Tratatto e reggimento utilissimo per seruirse nel tempo della peste de Giovanni Gieurot (Siena, Luca Bonetti, 1576) y Avvertimenti sopra la preservatione dalla peste de M. Antonio Minutoli (Luca, Vicenzo Busdraghi, 1576). El texto está plagado de anotaciones manuscritas. Encontraríamos dos tipos de letra con varios colores de tinta, lo que indica la existencia de múltiples anotadores; aspecto poco extraño dado el origen escolar del volumen. Siguiendo los trabajos de Crosby, Henry Ettinghausen, María José Alonso Veloso, Carlos Fernández González, Sofía Simões, Alfonso Rey, Luisa López Grigera, Isabel Pérez Cuenca y Lía Schwartz ${ }^{19}$, podemos destacar que muchas de las anotaciones manuscritas del volumen son, en efecto, de Quevedo.

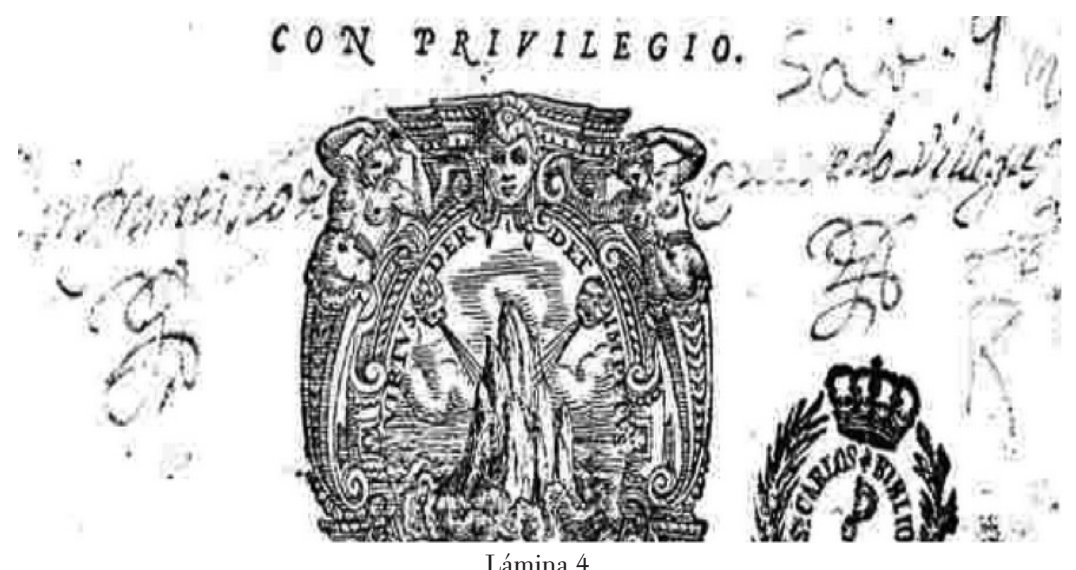

Ex libris de Quevedo en Libro della natura et virtù delle cose che nutriscono de Johannes Michael Savonarola, Biblioteca Histórica Marqués de Valdecilla, Universidad Complutense de Madrid, BH MED 1051(1)

Alfonso Rey, en su edición de la Virtud militante contra las cuatro pestes del mundo, parte de las normas de Félix del Val Latierro para su análisis de la letra de Quevedo y desarrolla una caracterización de la letra, aunque se centra solo en el manuscrito autógrafo del texto, lo que quizá impide que lo podamos tomar como referencia para estas anotaciones de carácter marginal. López Grigera, en el análisis más exhaustivo de la letra del Quevedo lector hasta la fecha, amplía lo dicho por Luis Astrana Marín, Crosby y Rey y mantiene que encontramos una diversidad de tipos de letra en Quevedo que dependen del fin anotativo o compositivo que persiga el señor de la Torre: una minúscula bastarda o itálica no cursiva, o itálica que no usa ligaduras más que alguna vez en sílaba. Asimismo, distingue entre letras cursiva y lo que ella denomina «libra-

19. Ettinghausen, 1964; Alonso Veloso, 2010b; Fernández y Simões, 2011, 2012; Rey, ed., 1985; López Grigera, 2002; Pérez Cuenca, 2003 y 2004 y Pérez Cuenca y Schwartz, 1999. 


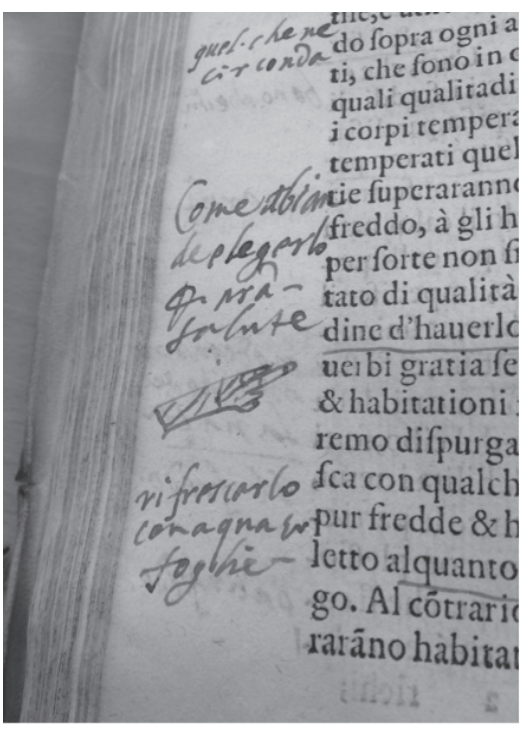

Manicula con angulación a la derecha de $45^{\circ}$; anotaciones al

Libro della natura et virtù delle cose che nutriscono de Johannes Michael Savonarola,

Biblioteca Histórica Marqués de Valdecilla, Universidad Complutense de Madrid, BH MED 1051(1)

cuaderno encontramos siete rúbricas que se asemejan enormemente a las que Quevedo utiliza para sellar sus ex libris manuscritos ${ }^{20} \mathrm{y}$ al final del segundo, una exacta a la de la portada $^{21}$. Estas rúbricas (no sería muy correcto hablar de filigranas) presentan las características de una probatio calami sive pennae (lámina 7). En realidad, ya efectuada la función legal de firmar el documento con una rúbrica (una "firma) que diríamos hoy en día), la probatio calami indica la prueba de la nueva pluma sobre los márgenes, espacios en blanco, folios de guarda o, como en este caso, en el verso de una pá- ria». Asimismo, varias de las anotaciones marginales coinciden con la caligrafía quevediana descrita por Pérez Cuenca y Schwartz como angulosa y con una suave inclinación hacia la derecha (72).

López Grigera indica que una de las improntas características de las anotaciones quevedianas en el interior del libro son, dentro del grupo de marcas y subrayados, unas «manos que apuntan». En el texto de Savonarola encontramos dos tipos de manecillas o manicula: una con angulación hacia la derecha, por ejemplo, la del folio $\mathrm{x} y$ otra con una angulación hacia la izquierda (láminas 5 y 6). En ambos casos se trata de subrayados. Al final del primer

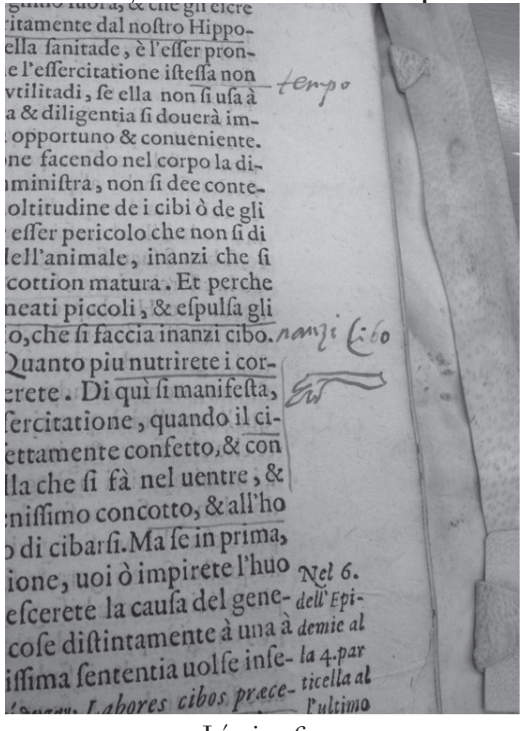

\section{Lámina 6.}

Manicula con angulación de $210^{\circ}$; anotaciones al Libro della natura et virtù delle cose che nutriscono de Johannes Michael Savonarola, Biblioteca Histórica Marqués de Valdecilla, Universidad Complutense de Madrid, BH MED 1051(1)

20. Savonarola, Libro della natura, fol. *4v.

21. Savonarola, Libro della natura, fol. $* * 4 \mathrm{v}$. 


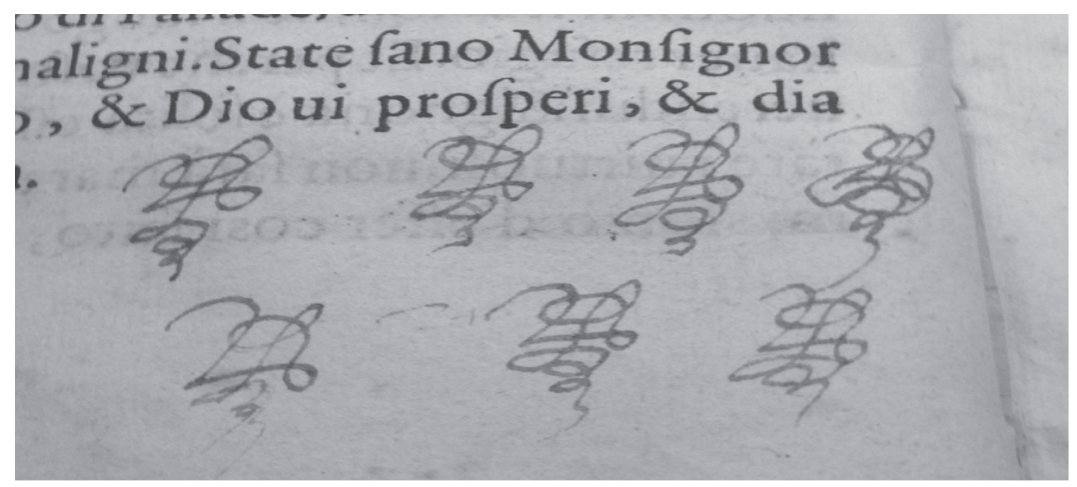

Lámina 7.

Probatio calami; anotaciones al Libro della natura et virtù delle cose che nutriscono de Johannes Michael Savonarola,

Biblioteca Histórica Marqués de Valdecilla,

Universidad Complutense de Madrid, вн MED 1051(1)

gina. Una mano (o más) calibra el cálamo; a veces la deja correr con despreocupación y llega a perfilar algún dibujo, en este caso la propia rúbrica de Quevedo. En apariencia, estos ensayos de escritura no hacen sino deteriorar el documento, pero nos otorgan detalles curiosos de las prácticas scribendi de Quevedo (si es que son de él puesto que algunos presentan trazos no usuales entre los del madrileño). De hecho, pese a las siete rúbricas de la probatio Quevedo (esta rúbrica ya es innegable) todavía tiene la necesidad de rubricar (lámina 8).

Un análisis caligráfico respalda la atribución. En las anotaciones «il de $\operatorname{coto}^{22}$ » y «virtudela bentosa ${ }^{23}{ }^{3}$, la preposición de es igual a las de los ex libris (lámina 9). En la anotación “Ingegno ${ }^{24}$ » la $g$ minúscula con cola volada es idéntica a las de los ex libris y a la de las anotaciones que realizó Quevedo a la Poética de Aristóteles, tan magníficamente estudiadas por Sánchez Laílla (láminas Universidad Complutense de Madrid, Bн MED 1051(1)

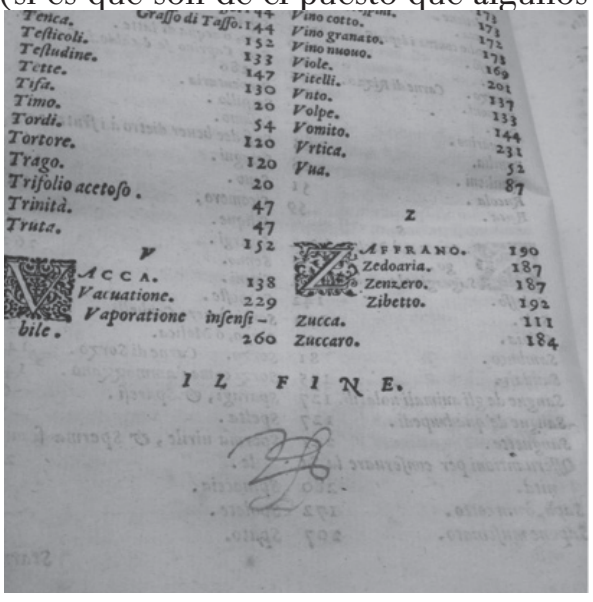

Lámina 8.

Rúbrica. Anotaciones al Libro della natura et virtù delle cose che nutriscono de Johannes Michael Savonarola, 10, 11 y 12). De igual modo,

22. Savonarola, Libro della natura, fol. 65.

23. Savonarola, Libro della natura, fol. 68.

24. Savonarola, Libro della natura, fol. 158. 


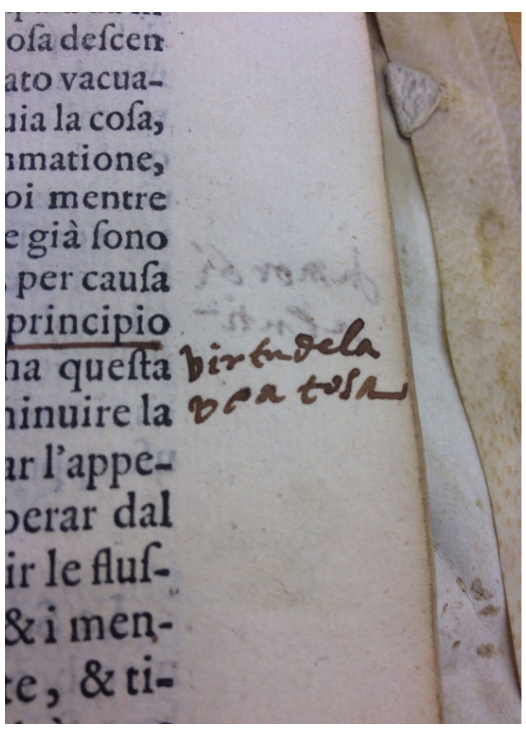

Lámina 9.

Preposición $d e$; anotaciones al

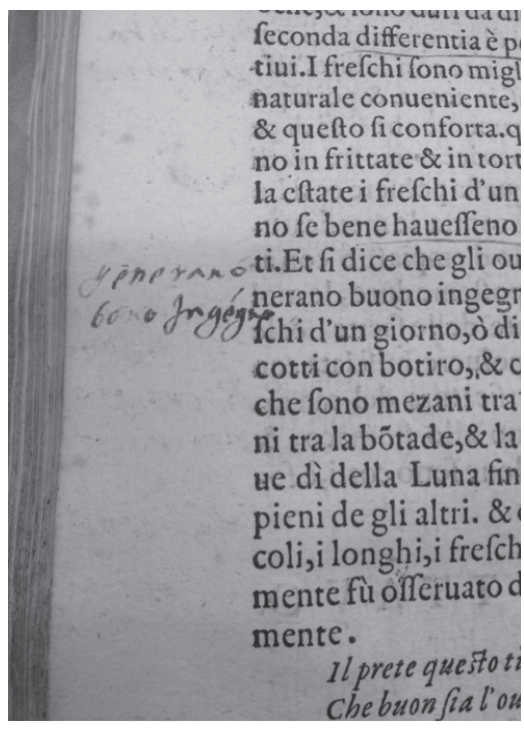

Lámina 10.

Anotación Ingegno (158); anotaciones al

Libro della natura et virtù delle cose che nutriscono Libro della natura et virtù delle cose che nutriscono de Johannes Michael Savonarola, de Johannes Michael Savonarola,

Biblioteca Histórica Marqués de Valdecilla, Universidad Complutense de Madrid, BH MED 1051(1)

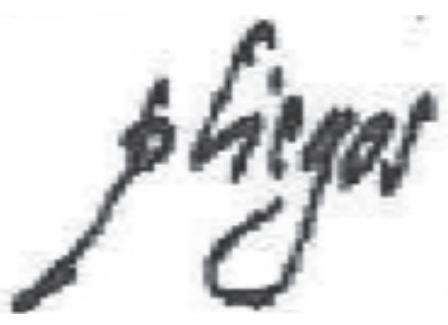

Lámina 11.

Anotación pliegos ms. 21883, fol. 12

(López Grigera 2002).
Biblioteca Histórica Marqués de Valdecilla,

Universidad Complutense de Madrid, BH MED 1051(1)

ming um te for cosion ciensetierieporivina rremternite, inafietie nearingu wo netoros ber.

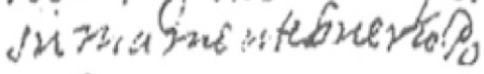
restuieres refentafin conntiat in Scateitos cayne lis "ientes nobre

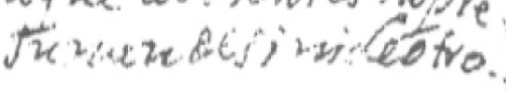

Lámina 12.

Anotación al margen de la Poética de Aristóteles, fol. 45

(Sánchez Laílla 2003).

Nótense la $g$ minúscula y la preposición $d e$. 


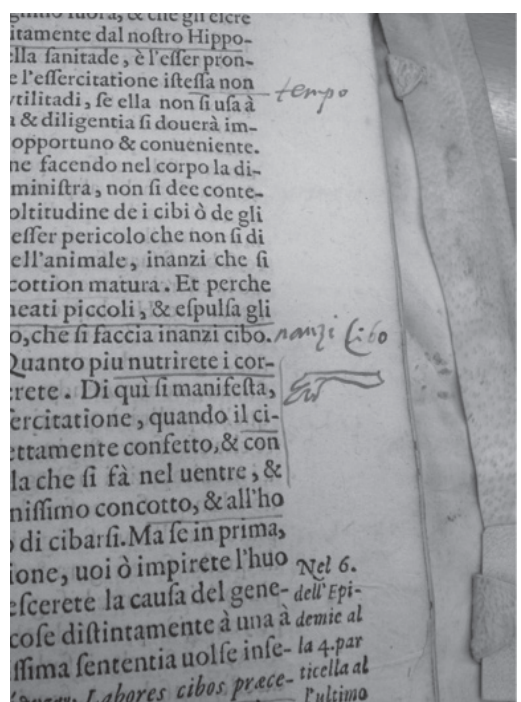

Lámina 13.

$C$ mayúscula»; anotaciones al

Libro della natura et virtù delle cose che nutriscono de Johannes Michael Savonarola,

Biblioteca Histórica Marqués de Valdecilla, Universidad Complutense de Madrid, BH MED 1051(1)

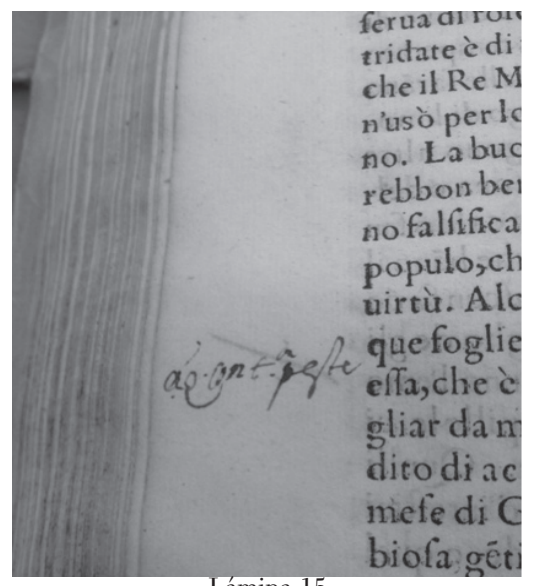

Lámina 15

$q$ minúscula con cola volada hacia la derecha; anotaciones al

Libro della natura et virtù delle cose che nutriscono de Johannes Michael Savonarola,

Biblioteca Histórica Marqués de Valdecilla, Universidad Complutense de Madrid, BH MED 1051(1)

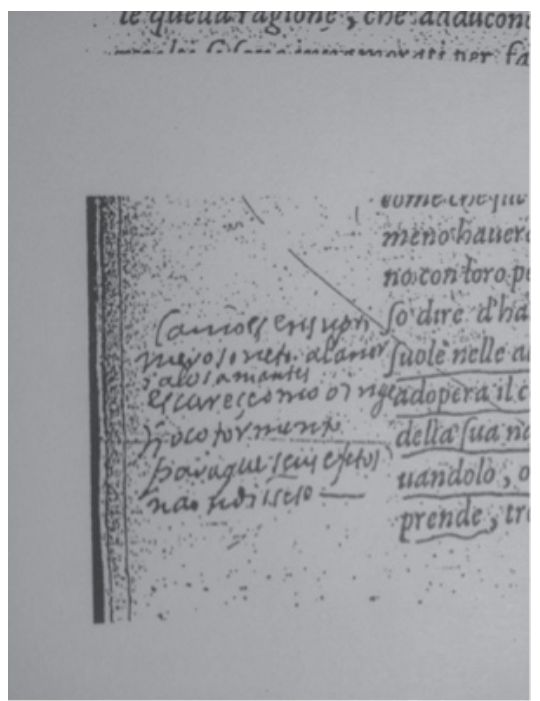

Lámina 14.

$C$ mayúscula», López Grigera, 2002, p. 191.

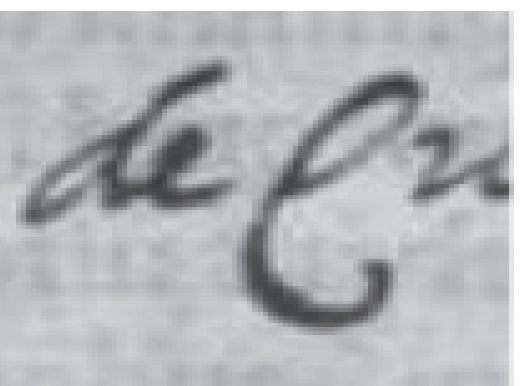

Lámina 16.

Ejemplo de $q$ minúscula con cola volada hacia la derecha en la firma de De octo partibus or [ati]onis lib. I de Constantino Lascaris,

Biblioteca Histórica Marqués de Valdecilla, Universidad Complutense de Madrid, BH FLL Res.306 


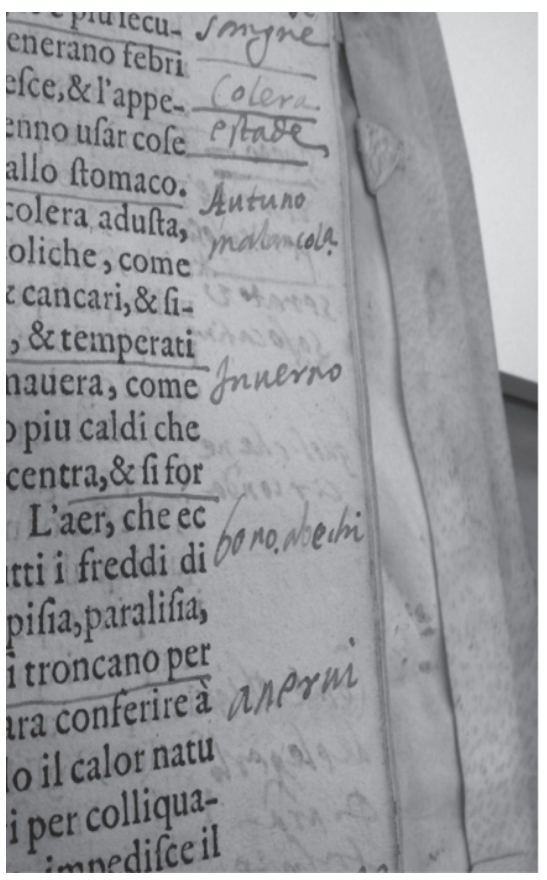

Lámina 17.

Imayúscula; anotaciones al

Libro della natura et virtu delle cose che nutriscono de Johannes Michael Savonarola,

Biblioteca Histórica Marqués de Valdecilla, Universidad Complutense de Madrid, BH MED 1051(1) la $C$ mayúscula que sobresale por arriba y por abajo y que abraza la minúscula siguiente es otra de las letras características de Quevedo, la encontramos en las anotaciones el Tratatto dell'amore umano de Flaminio Nobili (Lucca, 1567, BL Ms. 12108) cuya letra es «indudable» para López Grigera ${ }^{25}$ (láminas 13 y 14). Asimismo, la $q$ minúscula con una cola volada hacia la derecha es exacta a las de las firmas de los ex libris. Esta $q$ es tan característica de la letra de Quevedo que es igual en mayúsculas, minúsculas, cursivas y lo que López Grigera denomina «librarias» (lámina $15 \mathrm{y}$ 16). Asimismo, la I mayúscula de las anotaciones coincide con los modelos expuestos por López Grigera (láminas 17 y 18).

Por las características de la letra del impreso de Savonarola, me atrevería a indicar que, de ser de la pluma quevediana, se trata de un texto anotado a partir de los años 20. López Grigera, a partir de un corpus de 16 anotacio-

nes indudables y dos dudosas establece una cronología de las anotaciones autógrafas de Quevedo entre los grupos de La España defendida (1613), el papel autógrafo en que enumera sus antepasados para el ingreso en la orden de Santiago (1617), las anotaciones de la Retórica de Aristóteles (h. 1624), los de la Virtud militante, y

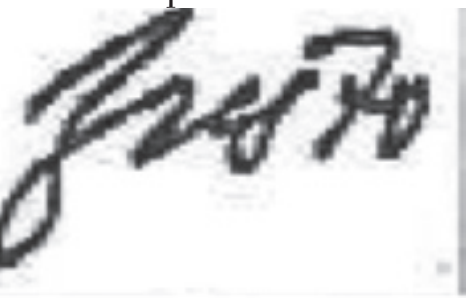

Lámina 18.

I mayúscula epístolas Sancho de Sandoval (de los años 30), el Martirio pretensor del mártir (1640) y la Providencia de Dios de 1641. Así lo indica precisamente una de las letras que escoge López Grigera como referencia, la $z$ minúscula, la cual «sobresale por arriba 
y abajo del renglón, crece en ancho y se hacen enzarzados sus trazos horizontales» ${ }^{26}$. Los ejemplos escogidos coinciden con los comentarios al Eliano, tan magistralmente estudiados por Pérez Cuenca y Schwartz ${ }^{27}$ (láminas 19 y 20). A continuación, presento una breve cala de los ejemplos atribuibles a Quevedo en el Libro della natura et virtù delle cose che nutriscono $\&$ delle cose non natura$l i^{28}$ :

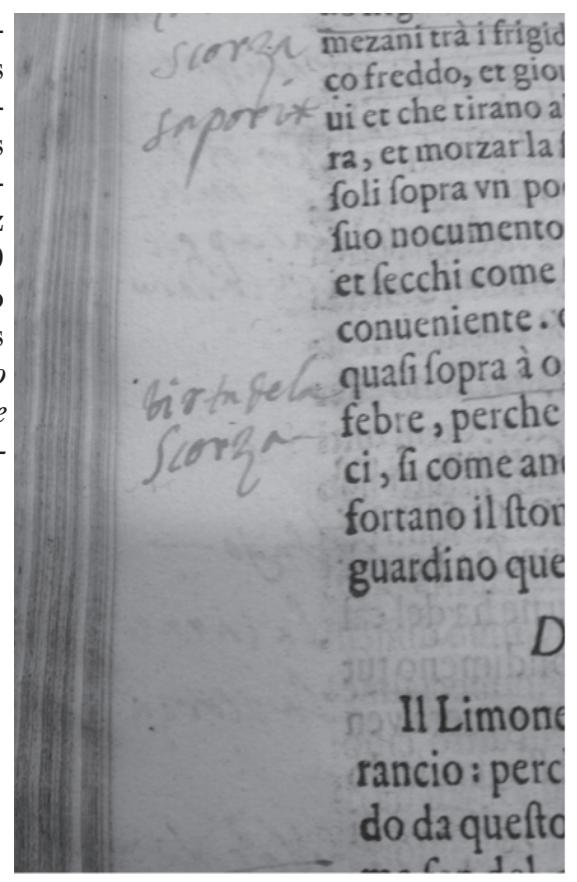

Lámina 19. $z$ minúscula. Lámina 12. $q$ minúscula con cola volada hacia la derecha.

Anotaciones al Libro della natura et virtù delle cose che nutriscono de Johannes Michael Savonarola, Biblioteca Histórica Marqués de Valdecilla, Universidad Complutense de Madrid, BH MED 1051(1)

pulici-morino

De la cicorea

(46) prugno

porcino

Della portulaca

(46) Emoroide

Del arancio

(92) sugo / carne

scorza

sapori

virtù de la / scorza

Del cigno

(126) cigni astrolog

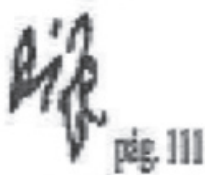

Lámina 20.

Anotaciones al Eliano

(Pérez Cuenca y Schwartz 76)

26. Pérez Cuenca y Schwartz, 1999, pp. 167-168.

27. Pérez Cuenca y Schwartz, 1999.

28. Evitamos la repetición bibliográfica (Savonarola, Libro della natura) en nota. 


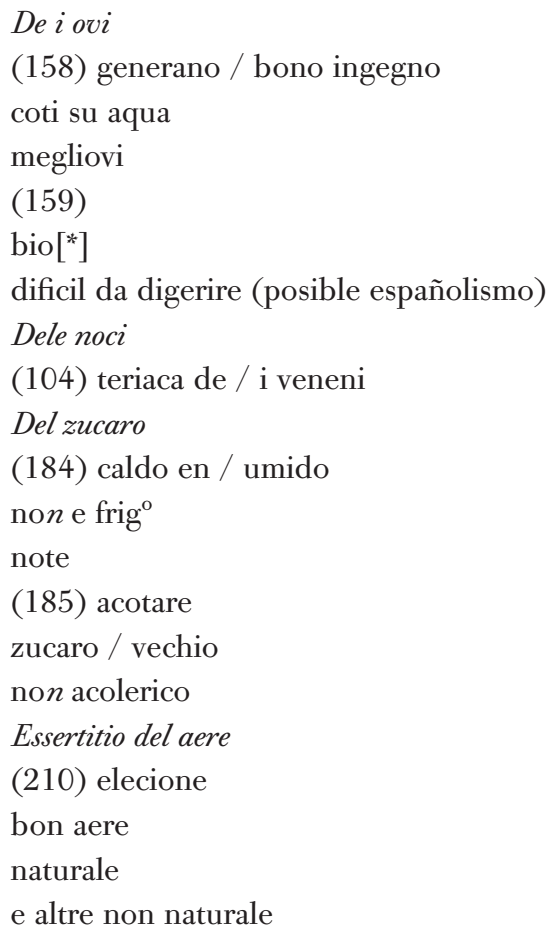

A la vez las notas atribuibles en el Tratatto e reggimento utilissimo per servirsene nel tempo della peste de Giovanni Gieruot (Siena, Luca Bonetti, 1576) serían en Delle medicine preservative

(16) aq cont ${ }^{\mathrm{a}}$ peste (aqua contra peste)

El modelo anotativo parece responder a dos intereses contrapuestos. Por un lado, encontramos subrayados y comentarios de carácter médico que responden bien a lo que Michael Solomon denominara el sickly reader, aquel que busca un remedio y anota buscando una solución puntual ${ }^{29}$. Parecería que tiene interés en las propiedades del azúcar o las cáscaras (scorza); por otro lado, encontramos unas anotaciones eruditas, que presentan interés en curiosidades como que los cisnes son buenos astrólogos, que los huevos otorgan gran ingenio, que las nueces sirven como triaca del veneno o que el agua es buena contra la peste.

En resumen, parece que la autoría de Quevedo es más que probable como lo demuestra una comparación entre los ejemplos escogidos de letra quevediana en este ejemplar y aquellas que se han testado anteriormente en los estudios que preceden a este. Por ejemplo, la pre-

29. Solomon, 2002. 
posición de y la g minúscula son iguales a las de las anotaciones al ms. 21883 (fol. 12) y a la Poética de Aristóteles (fol. 45); la $q$ minúscula con cola volada hacia la derecha es la de los ex libris mencionados; la $C$ y la $I$ mayúsculas son exactas a las de las calas efectuadas por López Grigera $^{30}$, y la $z$ minúscula comparada con la de las anotaciones al Eliano realizadas por Schwartz y Pérez Cuenca ${ }^{31}$. De momento, los ejemplos presentes bastan para demostrar la lectura que realizó Quevedo de este texto y la importancia que en su formación tendría ${ }^{32}$. Podríamos concluir que estos hallazgos complutenses son importantes en diversos niveles. Los volúmenes provenientes de la colección de la Facultad de Filología y, anteriormente, del colegio Imperial de los Jesuitas (el Lascaris y el Driedo), funcionan como fuentes, directas la una, indirecta la otra, de textos quevedianos. El primero es, sin duda, el ejemplar que utilizó Quevedo para su traducción del seudo-Focílides; el segundo es un ejemplar utilizado para las obras morales. Las obras provenientes de la colección de la Facultad de Medicina y, en última instancia, del Instituto de Salud San Carlos, son igualmente interesantes. El de Vito y el Savonarola muestran un interés claro en temas médicos como la nutrición y la extensión de la peste, a la par que permiten complementar los conocimientos gnómicos del madrileño. La diversa procedencia de los ejemplares nos permite, asimismo, analizar la evolución de sus prácticas notandi, pues pasamos de unos textos poco anotados antes de 1613 a unos muy anotados posteriormente. Hombre de su tiempo, Quevedo era un erudito complejo con variados intereses de los que estos cuatro volúmenes son magníficos testimonios.

\section{Bibliografía}

Alonso Veloso, María José, «Quevedo en sus lecturas: anotaciones autógrafas y subrayadas en cuatro impresos de la Biblioteca de Menéndez Pelayo». Manuscrt.Cao, 8, 2010a.

Alonso Veloso, María José, “Quevedo, lector del Anticlaudiano de Alain de Lille. Noticia sobre nuevas anotaciones autógrafas», La Perinola, 14, 2010b, pp. 277-303.

Asensio, Eugenio, «El erasmismo y las corrientes espirituales afines (conversos, franciscanos, italianizantes)», Revista de Filología Española, xxxvi, 1952, pp. 31-99.

30. López Grigera, 2002, p. 191.

31. Schwartz y Pérez Cuenca, 1999, p. 76.

32. Esperamos complementar los hallazgos con una investigación basada en la fotografía hiperespectral, que planeamos ayudar a realizar en su momento junto a Alberto Montaner y la propia Biblioteca Marqués de Valdecilla. Posiblemente, contaremos con un proyecto ad hoc que desentrañe la autoría de las anotaciones de estos documentos. Para la técnica a utilizar, véanse los artículos de Montaner (2008 y 2009). Asimismo, las anotaciones piden, sin duda, un análisis de mayor profundización, que hacemos en un artículo. Ver Vélez-Sainz, en prensa. 
Astrana Marín, Luis, «Modo de escribir de Quevedo: su especial puntuación y ortografía», en Francisco de Quevedo, Obras completas, Madrid, Aguilar, 1945, pp. 1349-1352.

Balcells, José María, "Quevedo, traductor del griego», Scriptura, 4, 1988, pp. 35-41.

Cacho Casal, Rodrigo, "Quevedo y su lectura de la divina Commedia», Voz y Letra, 9, 2, 1998, pp. 53-75.

Cacho Casal, Rodrigo, “Algunas fuentes italianas sobre el Buscón», en Estudios sobre el Buscón, ed. Alfonso Rey, Pamplona. Eunsa, 2003, pp. 191-219.

Carilla, Emilio, Quevedo (entre dos centenarios), Tucumán, Universidad Nacional, 1949 , p. 69.

Castanien, Donald G., "Quevedo's translation of the Pseudo-Phocylides», Philological Quarterly, xL, 1961, pp. 44-45.

Crosby, James O., «La historia del texto de la traducción de Focilides», En torno a la poesía de Quevedo, Madrid, Castalia, 1967, pp. 175-204.

Driedo, Johannes, Ioannis Driedonis... De concordia liberi arbitrï, \& praedestinationis diuinae, liber unus, Louanii, ex officina Rutgeri Reseij, 1537.

Ettinghausen, Henry, «Quevedo Marginalia: his Copy of Florus' Epitome», Modern Language Review, 59, 1964, pp. 391-398.

Ettinghaussen, Henry, Francisco de Quevedo and the Neostoic Movement, Oxford, University Press, 1972.

Fernández Galiano, Manuel, “Notas sobre una oda incompleta de Quevedo», Revista de la Biblioteca, Archivo y Museo del Ayuntamiento de Madrid, 14, 1945, pp. 350-351.

Fernández González, Carlos y Sofía Simões, «Nuevas aportaciones a la biblioteca de Francisco de Quevedom, Manuscrt.Cao, 11, 2011.

Fernández González, Carlos y Sofía Simões, «Apéndice a Nuevas aportaciones a la biblioteca de Francisco de Quevedo», Manuscrt.Cao, 12, 2012.

Lascaris, Constantino, In hoc libro haec habentur Constantini Lascaris... De octo partibus or[ati]onis lib. I; eiusdem De constructione liber secundus; eiusdem De nomine [et] uerbo liber tertius; eiusdem De pronomine in omni idiomate loquendi, ac ut poetae utuntur opusculum... Cebetis tabula [et] graeca [et] latina... De literis graecis ac diphthongis... Abbreuiationes quibus... graeci utuntur; Oratio Dominica [et] duplex salutatio ad Beatiss. Virginem; Euangelium diui Ioannis Euangelistae; Carmina aurea Pythagorae; Phocylidis poema ad bene beateque uiuendum; De idiomatibus linguarum tres tractatus Ioannis grammatici, Eustathii, Corinthi cum interpretatione latina; Introductio perbreuis ad hebraicam linguam, Venetiis, apud Aldum, 1512.

López Grigera, Luisa, “Anotaciones de Quevedo lector», en El libro antiguo español, vi. De libros, librerías, imprentas y lectores, Salamanca, Universidad de Salamanca, 2002, pp. 163-191.

Maldonado, Felipe C. R. «Algunos datos sobre la composición y dispersión de la biblioteca de Quevedom, en Homenaje a la memoria de don Antonio RodríguezMoñino 1910-1970, Madrid, Castalia, 1975, pp. 405-428.

Maldonado, Javier, «Biblioteca de Quevedo», Manuscr.Cao, 7, 2011.

Maldonado, Javier, «Biblioteca de Quevedo 2», Manuscr.Cao, 12, 2012.

Martinengo, Alessandro, «La biblioteca de Quevedo», en La astrología en la obra de Quevedo, Madrid, Alhambra, 1983, pp. 173-179.

Montaner Frutos, Alberto, “The Medievalist's Gadget: Hyperspectral Photography and the Phantom Scribe», Ecdotica: Rivista de Studi Testuali, 2008, 5, pp. 359-375. 
Montaner Frutos, Alberto, «La fotografía hiperespectral y la restauración virtual de códices medievales: aplicación al manuscrito único del Cantar de mio Cid,, Los codices literarios de la Edad Media. Interpretación, historia, técnicas y catalogación, ed. Pedro Cátedra, Belén Carro Carbajal y Javier Durán Barceló, Logroño, Cilengua, 2009, pp. 261-281.

Pérez Cuenca, Isabel, «Las lecturas de Quevedo a la luz de algunos impresos de su biblioteca», La Perinola, 7, 2003, pp. 297-333.

Pérez Cuenca, Isabel, «Localización y descripción de algunos impresos de la biblioteca de Quevedom, en Actas del XIV Congreso de la Asociación Internacional de Hispanistas. II. Literatura Española, siglos XVI y XVII, Newark-Delaware, Juan de la Cuesta, 2004, pp. 447-465.

Quevedo, Francisco de, Obra poética, ed. Juan Manuel Blecua, Madrid, Castalia, 1985, 4 vols.

Quevedo, Francisco de, Virtud militante contra las cuatro pestes del mundo, ed. Alfonso Rey, Santiago de Compostela, Universidad de Santiago de Compostela, 1985.

Sánchez Laílla, Luis, "Quevedo al margen: tres notas a un comentario aristotélico», Bulletin Hispanique, 105, 2003, pp. 489-506.

Savonarola, Johannes Michael, Libro della natura et virtù delle cose che nutriscono E delle cose non naturali: con alcune osseruationi per conseruar la sanità $\&$ alcuni quesiti bellissimi da notare / raccolto... per M. Michel Sauonarola, Venetia, presso Domenico \& Gio. Battista Guerra, 1576.

Schwartz Lerner, Lía e Isabel Pérez Cuenca, «Unas notas autógrafas de Quevedo en un libro desconocido de su biblioteca», Boletín de la Real Academia Española, 79, 276, 1999, pp. 67-91.

Solomon, Michael, «The Sickly Reader and the Vernacular Text in Late Medieval and Early Modern Spain», en Two Generations: A Tribute to Lloyd A. Kasten (1905-1999), ed. Francisco Gago-Jover, New York, Hispanic Seminary of Medieval Studies, 2002, pp. 217-228.

Val Latierro, Félix del, Grafocritica: el documento, la escritura y su proyección forense, Madrid, Tecnos, 1963.

Vélez-Sainz, Julio, «Médicos, adivinos y profesores de secretos en la biblioteca de Francisco de Quevedom, Médicos, adivinos y profesores de secretos, ed. Folke Gernert, Toulouse, Pum, (en prensa).

Vito, Giovanni de, Ioannis de Vito... De causis nostrarum calamitatum et de morbis epidemicis: qui vulgabantur per totum regnum Neap. anno d[omi]ni 1600. Cum pronosticis vsq[ue] ad annum 1608, Neapoli, apud Io. Iacobum Carlinum typographum Curiae Archiepiscopalis, 1602. 

Reseñas 
